\title{
Real Options - Realistic Valuation
}

\author{
Marek Capiński and Wiktor Patena
}

\author{
Marek Capiński, Ph.D. \\ Department Chair and Professor of Finance at Nowy Sacz School of Business - National Louis University, \\ Zielona 27, 33-300 Nowy Sacz, Poland \\ capinski@wsb-nlu.edu.pl, tel. +48 184499288 \\ Wiktor Patena, Ph.D. \\ Assistant Professor of Finance at Nowy Sacz School of Business - National Louis University, Zielona 27, 33- \\ 300 Nowy Sacz, Poland \\ patena@wsb-nlu.edu.pl, tel. +48 184499288
}

\begin{abstract}
In valuation of real options, a widely accepted assumption is that the underlying real asset is perfectly correlated with a financial one. As a result, valuation techniques from the financial world can be used. Since this assumption is in general unrealistic and may lead to substantial mispricing, even if the correlation is very high but not perfect, we argue that a different approach is more adequate. It is based on a simple principle of invariance of the market price of risk computed for certain portfolios involving the underlying asset and the options. This is illustrated on a simple model where one can see the relations between the real option prices and the correlation.
\end{abstract}

Key words: finance, real options valuation, replication 


\section{Introduction}

We begin with a brief presentation of the basic ideas and examples presenting the concept of real option. If a company conducts market research, it in fact buys an option: if the research is fruitful the company will launch the product. The expenses incurred during the market research phase represent the option premium, and the cost of further investment incurred at some future time $T$ is the exercise price. The payoff depends on the success of the new product and it is the difference between the value of the future expected cash flows (discounted to time $T$ ) and the investment. Obviously, it is zero when the result of the market research is negative - the company will not invest.

The example resembles a situation that has been known in the world of finance under the name of call option since the payoff is given by the following formula: $\max (0, R(T)-K)$, where $K$ is the value of the investment and $R(T)$ is the value at time $T$ of the generated cash flows.

On the other hand, put options may provide insurance against risk a company may face if in case of a failure and a necessity to reduce the production facilities.

Such options as they appear in real business activities are very specific, as the examples above show. According to Schwartz [University of Maryland 2003: 22] they fall into two categories. Some easily fit to the Black-Scholes world since their underlying assets (e.g.: oil, copper, gold deposits) have traded future contracts which can be used for replication - a sine qua non condition for Black-Scholes valuation method. Others (e.g.: the ones for Internet companies, $\mathrm{R} \& \mathrm{D}$ projects, real estate) can be rarely written or sold. We cannot hedge, nor can we replicate them using the underlying assets and bonds, since in such applications of real options it is impossible to find a financial asset perfectly correlated with the real one. These 
features distinguish them sharply from financial options. This raises the question how to valuate such real options. The question is fairly important since, once we master the valuation methods, we may properly use real options to value both individual projects and entire companies. However, if the limitations are not overcome, implementing real options, in Borison's opinion (University of Maryland 2003: 11), "will still face a lot of resistance".

If the real option can be related to a financial option, which is the case when there is a financial asset perfectly correlated with the underlying asset of the real option, then applying the valuation tools developed for financial options is justified. However, we face a danger of mispricing if the correlation is not perfect. This is highlighted by a result of Hubalek and Schachermayer [Hubalek 2001] who prove (see Related Research section and Appendix 1) that by using the no-arbitrage principle nothing can be said about the price of a real option if a tradable (financial) underlying asset is not perfectly correlated with the real underlying.

This discovery led some (Henderson 2002, Schweitzer 1995) to discard pricing methods that use only the assumption of no arbitrage ${ }^{1}$, and consider a utility based approach instead. We tried to dispose of the subjectivity that is unavoidably related to the process of maximizing utility functions. Hence, in this paper still another approach is presented (although we consider our method of pricing in incomplete markets as the one belonging to the partial equilibrium category).

We build a modelling framework in which the starting point in solving the problem of pricing real options is still the assumption of no arbitrage, enriched by adding an invariant market price of risk assumption. The Sharpe index (compare: Cochrane, Sao-Requejo 1996) allows

\footnotetext{
${ }^{1}$ The assumption that the payoff of a real option can be replicated by means of financial securities is stronger than the assumption that the market is complete (which says that any claim contingent on financial assets can be replicated by means of them). This is because the real option is a claim on a non-traded asset. The completeness principle is often criticised as too restrictive and much recent effort has been put into option pricing in incomplete markets. Two main lines of research are: partial equilibrium and general equilibrium methods [Boyle 2001].
} 
selecting only one from many martingale measures. The new combination of assumptions leads to promising results that cast new light on how to price real options. Furthermore, the method, mostly due to its simplicity, can be easily implemented.

This paper will consider a specific real options problem. The problem is twofold; either the underlying asset is non-tradable or trading of the asset is not permissible. In either case, one needs to use another (but traded) asset in order to price the option (or hedge the payoff).

Let us analyze the first situation only (the other is less common - it applies only to executive stock options). Suppose one has a real business and wants to invest into a real option. Thus, we would have a portfolio composed of the real asset and real option. The question that arises is how much the option is worth. If we were able to find a financial asset perfectly correlated with the business there would be no need to price the option. However, finding such an asset is not an easy task.

There are two solutions to the problem we put forward.

1 For the business people the payoff is what matters. Hence, instead of investing into a real option the business person may as well consider buying a financial option if only the payoff it promises is identical to the one expected from the real option.

2 In order to price a real option one needs to consider a writer (issuing party). Since they do not exist (for real options), we create a hypothetical writer who is short the real option and to hedge goes long in a financial one. Since this writer is ourselves (or a financial company we have created), we end up having the real asset and the financial option. 
Both scenarios lead to the situation that we have a portfolio of the real asset and the financial option. At this point, one has to accept the fact that the value of the financial option (that is closely related to the real one) is so far the only benchmark we can refer to.

Another principle we adopt is this: from the point of view of a manager, acquiring a real option should not change the balance between the risk and the expected return. Hence, we propose the assumption that the market price of risk (defined as the expected return of an asset minus the risk free rate, divided by the standard deviation of the asset) for the business with the option should not be higher than the market price of risk for the business itself. This assumption underlies many applications of asset-pricing theory to corporate finance, including Cochrane (Cochrane, Sao-Requejo 1996) ${ }^{2}$ and Bielecki’s (Bielecki, Jeanblanc, Rutkowski $2003)^{3}$ approach - just to name a few. This is also motivated by the well-known fact that the market prices of risk for a derivative security and the underlying asset coincide for the single step binomial model. In other words, suppose the market is complete and perfect replication is possible. Assume that the price of an option is such a number that the resulting market price of risk is the same as for the underlying. Then this price is the same as the price obtained by the replication technique (the expectation of discounted payoff taken with respect to the risk neutral probability). Another consistency argument is that underlying is the call option on itself with zero strike price. It can be clearly seen that in the model below the price of this "option" coincides with the price of the underlying.

\footnotetext{
${ }^{2}$ In Cochrane and Sao-Requejo's paper the Sharpe index is the departure point for valuing uncertain payoffs. They "use a little economics (Sharpe ratio) to restrict the range of possible stochastic discount factors. The restrictions result in bounds on an asset price given the prices of a set of basis assets that can be used as approximate hedges". Thus, they find option prices via the minimum volatility of the discount factor (that corresponds to maximum Sharpe ratio).

${ }^{3}$ Recently, Bielecki, Jeanblanc and Rutkowski 2003, tackled the issue of pricing a derivative security in an incomplete market in the context of credit risk. Their approach is very similar to ours in that they add the derivative security to the underlying one thus forming a portfolio. This portfolio is analyzed by mean-variance considerations and the price of the derivative is postulated to be consistent with increase in final total wealth accompanied with decrease of total risk (standard deviation). Our approach differs since we consider the excess return instead of the additional wealth.
} 
It is widely accepted by economic theory that maximizing Sharpe index is a reasonable criterion for constructing portfolios. Our assumption is that adding a real option should not decrease this index, which is thus regarded as a benchmark. In doing so we take the view that the specific risk is a crucial ingredient. Market price of risk is concerned with risk measured as standard deviation which can be decomposed into specific (business, diversifiable) risk and market risk. For a large class of investors the latter is what matters since they tend to keep diversified portfolios and do not regard exposure to specific risk as an excuse for additional claims. However, for small businesses it is otherwise, since the specific business risk is prevailing with very limited diversification resulting from the fact that typically all the funds are being tied in the business (see [Cotner, Fletcher 2000], for instance).

Having these two benchmarks (financial option price and market price of risk for the portfolio), we can use the financial asset, gauge its correlation with the non-traded asset and determine the option price so that the two assumptions are met. For this, (instead of the original which was composed of the real business and real option) a portfolio composed of the real asset and a financial option is needed.

In practice, we search through available financial options and find one whose underlying asset seems closely correlated with our business. The precise correlation is determined (via historical returns). Then the relevant portfolio is constructed and the two-assumption framework generates a price of the real option

In the perfect correlation (complete market) case the resulting option premium coincides with the one obtained by standard approaches - our method gives the same result as well-known 
financial tools. In fact, this particular case will be used as a motivation and a point of departure for more general analysis. By modifying the portfolios, for instance by allowing only partial involvement in the real options or introducing an outside option writer, we will obtain a range of option values for each correlation. This range of values can be interpreted as realistic option prices within a bid-ask spread which is quite natural in incomplete markets even without transaction costs.

This approach is in line with corporate finance valuation principle, where the physical probability is used to estimate the expected value which is then discounted using the cost of capital, that is, the rate reflecting the risk.

In this paper, we illustrate the issues concerned with valuation of real options assuming that both the underlying real asset and a financial one follow a binomial model. In order to describe their statistical relationship, a quadrinomial (a four-way split) model is employed. We consider a simplified (but quite general in some respect) version, where the relation between the option prices obtained by using various approaches can be clearly seen.

\section{Related Research}

The starting point for option valuation was the work of Black and Scholes [Black 1973] and Merton [Merton 1973] in pricing financial options. These were followed by a simplified, binomial approach presented by Cox, Ross and Rubinstein [Cox 1979]. Since then, vast literature on financial options has emerged. Financial option valuation in complete markets (e.g. in binomial and Black-Scholes models) is based on replication. Using risk-neutral valuation, where the option premium is the expected value of a discounted payoff with respect 
to the martingale probability, is the technique that is equivalent to the one based on replication. The uniqueness of the martingale probability is equivalent to the completeness of the market model considered.

The majority of research on real options is based on employing the techniques developed for financial options despite the fact that the underlying of the former is rarely traded. This approach is based on assuming a strong version of the completeness of the market. "The existence of a traded 'twin security' (or a portfolio of traded securities) that is perfectly correlated with the non-traded asset in complete markets is sufficient for real-option valuation", says Trigeorgis [Trigeorgis 1999]. His book, as well as a classical contribution of Dixit and Pindick [Dixit 1994], contains a comprehensive presentation of this line of research, followed recently by many papers, in particular Amram [Amram 2000], Boer [Boer 2000], Kellog [Kellog 2000] - to mention just a few.

Some authors realise that the twin-security principle is strong, possibly unrealistic, and develop some ways to deal with this.

Copeland [Copeland 2000: 94] proposes an assumption that the real underlying asset can be treated as a traded financial asset itself (this may be regarded as a special case of the twinsecurity principle so this approach is vulnerable to the same extent). But even if so, the short selling is impossible, thus replication of a put cannot be performed.

Still another approach was presented by Constantinides [Constantinides1978] who claimed that risk-neutral valuation is analogous to discounting certainty-equivalent cash flows at the risk-free rate. Thus, any option on an asset (traded or not) can be valued by replacing its 
expected cash flows with the certainty equivalent cash flows and then behaving as if the world were risk neutral. However, it is not clear how to find the certainty equivalent cash flows.

Amram and Kulatilaka [Amram 2000] restrict the notion of real options defining them to be "the subset of strategic options in which the decision to exercise the option is basically determined by financial instruments or assets traded on the markets". However, most of the real options do not fit this definition.

In 2001 Fernandez [Fernandez 2002: 532] stated that "if the real options cannot be replicated, using financial option formulas for valuing real options is completely inappropriate". He suggests using a modified Black-Scholes formula to price a non-replicable call where, apart from standard inputs, the option's value also depends on the expected return $\mu$ of the real underlying and the discount rate $r_{K}$ that is appropriate for the project. This, however, is nothing else but the DCF method using physical probabilities to find the expected cash flow and the cost of capital for discounting. He does not propose any method for estimating this discount rate.

A rigorous and significant argument against using the financial tools to real options is provided by Hubalek and Schachermayer [Hubalek 2001]. They critically analyse the belief that when trying to valuate a real option a sufficiently good "surrogate asset" should do just as well as the underlying asset itself. The main result of the paper is that if the "surrogate asset" is not perfectly correlated to the real underlying then "for a European call option written on the real underlying one can not conclude anything on its price by using only no-arbitrage arguments: if only trading in the surrogate asset was allowed, then any number in $(0, \infty)$ is a possible price for this option without violating the no-arbitrage principle" (The theorem was 
formulated and proved for Black-Scholes model but since our paper deals with discrete models only, we present in the Appendix an exposition of the one step binomial counterpart of their result).The conclusions of [Hubalek 2001] reinforce Fernandez' opinion. As the authors say "the message of this theorem is not that applying the Black-Scholes methodology to real options is not correct, but: whenever one applies the Black-Scholes methodology to real options whose underlying is not a traded asset, one must be very careful and one has to be aware that preferences, subjective probabilities etc. have to come into play".

\section{Valuation}

\subsection{Model of asset dynamics}

We consider two assets, a real and a financial one, with prices for one-time step denoted by $X(n)$ and $Y(n)$, respectively, for $n=0,1$. We assume that $X(0)=Y(0)=100$. Each of the prices is assumed to follow a binomial model with returns $u=0.2, d=-0,05$ in a single step, the same for each asset. The risk free rate is assumed to be $r=0.05$.

To capture a general behaviour of the couple we need a quadrinomial (four-split) model, namely we allow four scenarios in a single step.

\begin{tabular}{|l|l|l|l|l|l|}
\hline Scenarios & $\boldsymbol{X}$ & $\boldsymbol{Y}$ & Probabilities & $\boldsymbol{X}(\mathbf{1})$ & $\boldsymbol{Y}(\mathbf{1})$ \\
\hline 1 & $\uparrow$ & $\uparrow$ & $p_{1}$ & 120 & 120 \\
\hline 2 & $\uparrow$ & $\downarrow$ & $p_{2}$ & 120 & 95 \\
\hline 3 & $\downarrow$ & $\uparrow$ & $p_{3}$ & 95 & 120 \\
\hline 4 & $\downarrow$ & $\downarrow$ & $p_{4}$ & 95 & 95 \\
\hline
\end{tabular}

Suppose that the probability distributions of $X, Y$ are given and for simplicity assume probability 0.5 for each movement. This imposes some restrictions on the numbers $p_{i}$ (apart 
from non-negativity): $p_{1}+p_{2}=0.5, p_{3}+p_{4}=0.5, p_{1}+p_{3}=0.5, p_{2}+p_{4}=0.5$, resulting in $p_{1}=p_{4}, p_{2}=p_{3}$. Hence the probabilities are as below - they are determined by a single parameter $q \in[0,0.5]$ :

\begin{tabular}{|l|l|l|l|l|l|}
\hline Scenarios & $\mathbf{X}$ & $\mathbf{Y}$ & Probabilities & Asset X & Asset Y \\
\hline 1 & $\uparrow$ & $\uparrow$ & $q$ & 120 & 120 \\
\hline 2 & $\uparrow$ & $\downarrow$ & $0.5-q$ & 120 & 95 \\
\hline 3 & $\downarrow$ & $\uparrow$ & $0.5-q$ & 95 & 120 \\
\hline 4 & $\downarrow$ & $\downarrow$ & $q$ & 95 & 95 \\
\hline
\end{tabular}

Despite this simplification, we shall be able to illustrate the crucial issues involved in pricing and hedging real options. One of the advantages of the model is that we can easily relate the correlation between $X$ and $Y$ to the value of $q$; for example, the correlation coefficient ${ }^{4} \rho$ is 1 for $q=0.5$ at one extreme, and $\rho=-1$ for $q=0$ at the other. In general, $\rho=4 \times q-1^{5}$. In fact, this model is general under the assumption that probabilities for $X$ and $Y$ are specified (by replacing 0.5 with the corresponding probability, the above setting can be easily modified).

\subsection{The case of perfect correlation}

Consider a put option written on the real asset $X$ with exercise price $K=100$ and exercise time $T=1$. In the case in question, where $\rho=1$, the values of both assets rise or fall simultaneously. However, we will use options written on financial assets $Y$ to form hedge portfolios and derive adequate real option prices in the context of imperfect correlation.

\footnotetext{
${ }^{4}$ One may wonder why we cannot find a perfectly correlated financial asset if we can determine the exact correlation between any two assets. Transitivity property should make finding a perfectly correlated portfolio a straightforward task, which obviously is true. However, the paper is focused solely on illustrating pricing errors stemming from imperfect correlation and numbers representing correlation degree in the paper are just examples.

${ }^{5}$ This can be obtained from a general correlation coefficient formula: $\rho_{X Y}=\frac{\operatorname{Cov}(X Y)}{\sigma_{X} \times \sigma_{Y}}$, where

$\operatorname{Cov}(X Y)=\sum_{i=1}^{n}\left(k_{X i}-\hat{k}_{X}\right)\left(k_{Y i}-\widehat{k}_{Y}\right) q_{i}$ and $k, \hat{k}$ are respectively realized and expected rates of return.
} 
The value of the put option on $Y$ can be computed easily. To replicate the payoff we need to sell the number $\Delta$ of shares of $Y$ and borrow the amount $B$ of funds (at the risk-free rate). The value of the portfolio constructed at the end of the period will be either $\Delta \times Y \times(1+u)+B \times(1+r)=0$ or $\Delta \times Y \times(1+d)+B \times(1+r)=5$. From these equations, the values $\Delta=-0.2$ and $B=22.857$ can be easily obtained, hence the option premium equal to the initial cost of constructing the hedge is $\Delta \times Y+B=2.857$. (Alternatively, we can use the martingale probability $p=\frac{r-d}{u-d}=0.4$ to find $\frac{p \times 0+(1-p) \times 5}{1+r}=2.857$.)

Since the correlation is perfect, the no-arbitrage principle guarantees that the real put has to be worth exactly the same as the financial one (put on $X$ is worth the same as the put on $Y$ ).

To illustrate the method we shall apply later on in a general case, we approach the problem from a slightly different angle; namely, we value a real put indirectly by forming a portfolio $P_{1}$ consisting of $X$ and the put on $Y$. In the present case we know that the portfolio is worth 102.857 and it gives either 120 or 100 at the end of the period (the put on $Y$ pays the additional 5 if $X$ is worth 95).

The same valuation can be applied to a call. The call on $Y$ (with the same exercise price) is worth 7.619. (We can use the martingale probability $p=0.4$ to find $\frac{p \times 20+(1-p) \times 0}{1+r}=7.619$.) A portfolio $P_{2}$ consisting of $X$ and the call on $Y$ is worth 107.619 at the beginning and pays either 140 or 95 at the end of the period.

Now, we compute the expected return and the risk, measured as the standard deviation, for both investments and compare them with $X$ : 


\begin{tabular}{|l|l|l|l|}
\hline & $\boldsymbol{X}$ & $P_{1}=\boldsymbol{X}+$ put on $\boldsymbol{Y}$ & $\boldsymbol{P}_{2}=\boldsymbol{X}+$ call on $\boldsymbol{Y}$ \\
\hline Expected return & $7.5 \%$ & $6.9 \%$ & $9.18 \%$ \\
\hline Standard deviation & $12.5 \%$ & $9.72 \%$ & $20.91 \%$ \\
\hline
\end{tabular}

It is obvious that the market price of risk is the same for $X, Y, P_{1}$ and $P_{2}$ and is equal to 0.2 . Recall that the market price of risk is defined as the excess return per unit risk, i.e. the difference between the expected return and the risk free rate divided by the standard deviation:

$$
\lambda=\frac{k_{P_{1}}-r}{\sigma_{P_{1}}}=\frac{k_{P_{2}}-r}{\sigma_{P_{2}}}=\frac{k_{X}-r}{\sigma_{X}}=0.2 .
$$

\subsection{General case}

The fundamental concept behind our approach to valuate the real options is that the market price of risk for the hedge portfolio (the option on $Y$ plus the $X$ asset) should be at least the same as the one for $X$ alone.

In the particular case of perfect correlation the Sharpe index is the same for the underlying and any option, so it is natural to require the same in a general situation of arbitrary correlation, bearing in mind the fact that in the special case the theories must meet.

Therefore, this is also true for the case of perfect correlation, as the above example shows. We then decide that the market price of risk is the criterion for valuation in a general case.

Consequently, we find the initial values of the portfolios so that the market prices of risk for both $P_{1}$ and $\mathrm{X}$ or $P_{2}$ and $X$ are identical and then, subtracting the initial value of $X$, we obtain the values of real put or call. The real option price can also be regarded as the reservation price - the amount an investor requires so she is indifferent between possessing a real asset and the portfolio composed of the real asset and the option. 
Remark. Note that from the buyer's point of view, acquiring a real option should not increase the risk and (1) would be then only a border-line case. However, the same is true for the option writer. The two requirements converge only if (1) is assumed.

In the case of perfect positive correlation the quadrinomial model yields (as shown in the table below) the same option prices as the replication method. For such prices the model is in equilibrium - the market price of risk equals 0.2 .

\begin{tabular}{|l|l|l|l|l|l|}
\hline Scenarios & $\mathbf{X}$ & $\mathbf{Y}$ & Probabilities & $P_{1}=\boldsymbol{X}+$ put on $\boldsymbol{Y}$ & $P_{2}=\boldsymbol{X}+$ call on $\boldsymbol{Y}$ \\
\hline 1 & $\uparrow$ & $\uparrow$ & 0.5 & 120 & 140 \\
\hline 2 & $\uparrow$ & $\downarrow$ & 0 & 125 & 120 \\
\hline 3 & $\downarrow$ & $\uparrow$ & 0 & 95 & 115 \\
\hline 4 & $\downarrow$ & $\downarrow$ & 0.5 & 100 & 95 \\
\hline Expected return & & & & $6.94 \%$ & $9.18 \%$ \\
\hline Standard deviation & & & & $9.7 \%$ & $20.9 \%$ \\
\hline Market Price of Risk & & & & $20 \%$ & $20 \%$ \\
\hline Implied Option Prices & & & & Put 2.857 & Call 7.619 \\
\hline
\end{tabular}

We also illustrate this approach for the correlation of 0.8 . The value of $q$ (derived from $\rho=4 \times q-1$ ) becomes 0.45 , and according to the principle assumed the put should be worth 2.742 and the price of the call should equal 7.836 .

\begin{tabular}{|l|l|l|l|l|l|}
\hline Scenarios & $\mathbf{X}$ & $\mathbf{Y}$ & Probabilities & $P_{1}=\boldsymbol{X}+$ put on $\boldsymbol{Y}$ & $P_{2}=\boldsymbol{X}+$ call on $\boldsymbol{Y}$ \\
\hline 1 & $\uparrow$ & $\uparrow$ & 0.45 & 120 & 140 \\
\hline 2 & $\uparrow$ & $\downarrow$ & 0.05 & 125 & 120 \\
\hline 3 & $\downarrow$ & $\uparrow$ & 0.05 & 95 & 115 \\
\hline 4 & $\downarrow$ & $\downarrow$ & 0.45 & 100 & 95 \\
\hline Expected return & & & & $7.1 \%$ & $8.96 \%$ \\
\hline Standard deviation & & & & $10.32 \%$ & $19.81 \%$ \\
\hline
\end{tabular}

The option value for different correlations can be easily found from the equation below

$$
0,2=\frac{k_{P_{1}}-0,05}{\sigma_{P_{1}}}=\frac{k_{P_{2}}-0.05}{\sigma_{P_{2}}},
$$


where $k_{P}, \sigma_{P}$ are expected return and standard deviation of portfolio $P$, respectively, and 0.2 is the market price of risk for $X$ (the formulae of the dependence of the option price on correlation can be found in Appendix 2, although in practice the prices are found with the help of VBA code and Solver function).

In Figure 1, it is easy to see that the value of the put increases (the graph is slightly convex) from 1.905 to 2.857 with the correlation going from -1 to 1 .

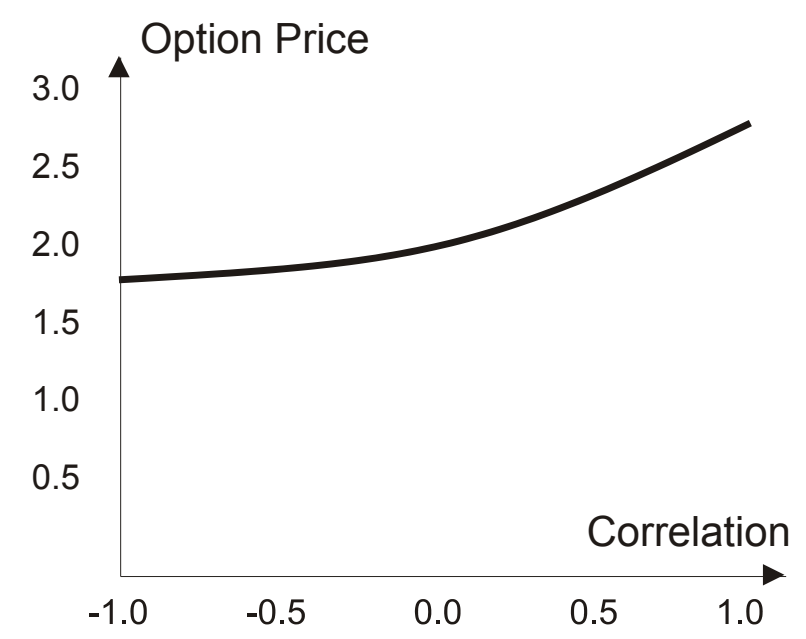

Figure 1. Value of the put as a function of correlation

We follow the same "market price of risk benchmarking" principle when calculating the price of a call. Paradoxically, the value of the call increases from to 7.62 to 11.43 as the correlation changes from 1 to -1 . Then we discover that in both cases the portfolio, apart from the real asset, has not one but two entwined components. The put that originally has payoffs of 0 and 5 turns into a call with payoffs of 5 and 0 (when correlation becomes -1) and the call with payoffs of 20 and 0 converts into a put with payoffs of 0 and 20. This can be explained by a different nature of financial and real options. For example, in scenario 3 in real world application, the business owner would not exercise the put option since this is tantamount to 
disposing of the company that, contrary to what the financial market "reckons", is far from going bankrupt.

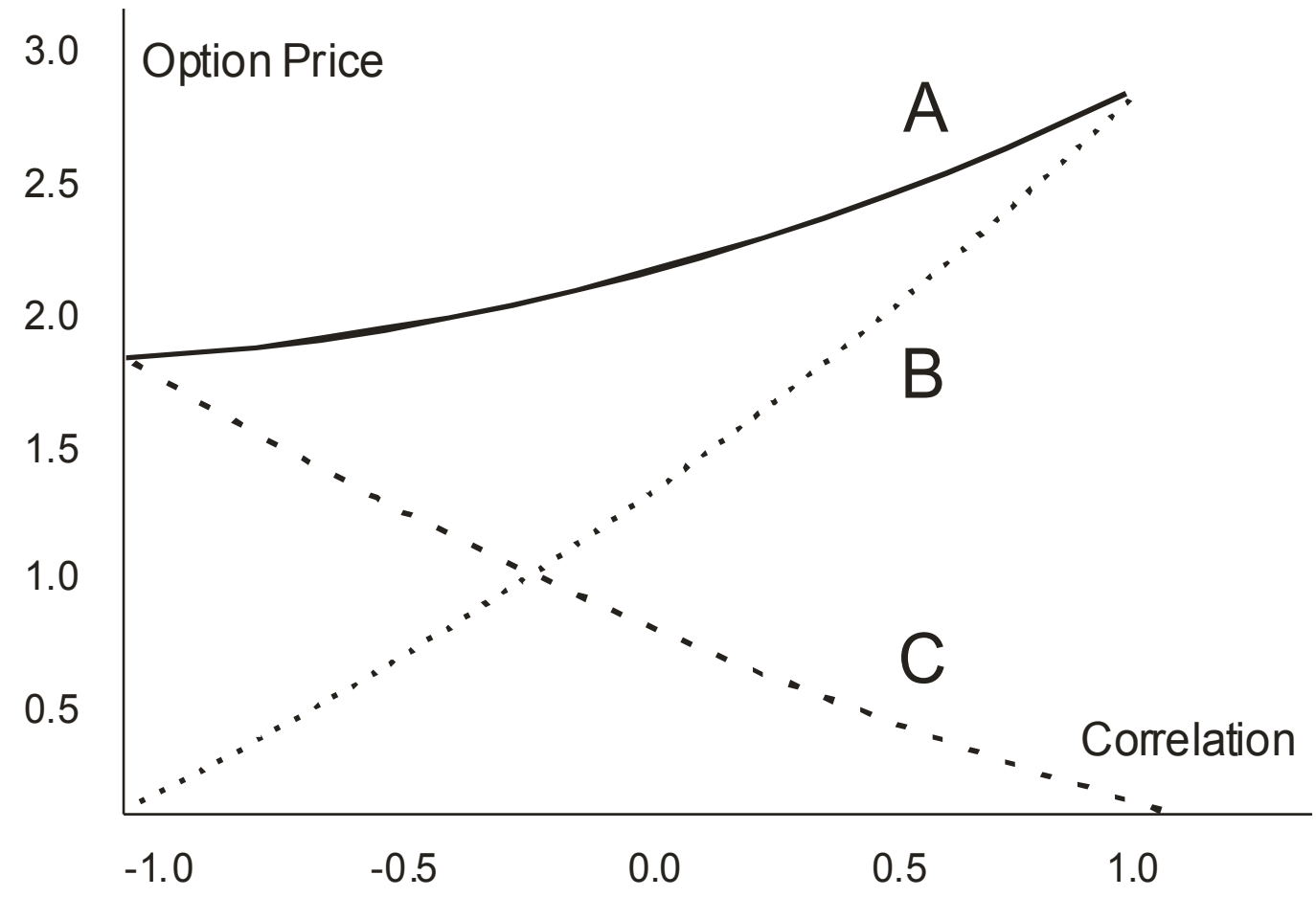

Figure 2. Decomposition of the Put

Thus, the B put in fact represents the real option, and its value can be found by subtracting the value of the call $\mathrm{C}$ from the put A (see Figure 2), that is removing the part of the payoff belonging to the call $\mathrm{C}$..

\begin{tabular}{|l|l|l|l|l|l|}
\hline Scenarios & $\mathbf{X}$ & $\mathbf{Y}$ & Probabilities & $P_{1}=\boldsymbol{X}+$ put on $\boldsymbol{Y}$ & $P_{2}=\boldsymbol{X}+$ call on $\boldsymbol{Y}$ \\
\hline 1 & $\uparrow$ & $\uparrow$ & 0.5 & 120 & 140 \\
\hline 2 & $\uparrow$ & $\downarrow$ & 0 & $125-5$ & 120 \\
\hline 3 & $\downarrow$ & $\uparrow$ & 0 & 95 & $115-20$ \\
\hline 4 & $\downarrow$ & $\downarrow$ & 0.5 & 100 & 95 \\
\hline
\end{tabular}

The value of the real put decreases (the graph is slightly convex) from 2.857 to 0 with the correlation going from 1 to -1 . The case of the call is handled in the same way - the value of the real call also falls from 7.619 to 0 . 
Figure 3 (also see the table below) shows possible pricing errors stemming from different values of correlation (the prices are scaled down to percentages). For example, changing the correlation from 1 to $0.8\left(\frac{0.2}{1-(-1)}=0.1\right.$, that is $10 \%$ change) results in $10.4 \%$ and $11 \%$ possible mispricing (defined as the percentage difference between financial option prices and the ones obtained by using our option pricing model) for the call and put prices respectively.

\begin{tabular}{|c|c|c|c|c|c|c|}
\hline \multicolumn{7}{|c|}{ Possible Pricing Errors } \\
\hline \multicolumn{7}{|c|}{ Put and call change within $(2.857: 0)$ and (7.619: 0$)$ ranges respectively } \\
\hline Correlation & 1 & 0.8 & 0.6 & 0.4 & 0.2 & -1 \\
\hline \multicolumn{2}{|c|}{ Correlation change } & $10 \%$ & $20 \%$ & $30 \%$ & $40 \%$ & $100 \%$ \\
\hline Put mispricing & 0.00 & 0.30 & 0.59 & 0.88 & 1.17 & 2.857 \\
\hline \multicolumn{2}{|c|}{ Put relative overestimation } & $10.4 \%$ & $20.6 \%$ & $30.8 \%$ & $40.9 \%$ & $100 \%$ \\
\hline Call mispricing & 0.00 & 0.84 & 1.67 & 2.49 & 3.29 & 7.619 \\
\hline \multicolumn{2}{|c|}{ Call relative overestimation } & $11.0 \%$ & $21.9 \%$ & $32.6 \%$ & $43.1 \%$ & $100 \%$ \\
\hline
\end{tabular}

The resulting error can be estimated as $(1-\rho)^{2}$, which is comparable to the result of Henderson and Hobson [Henderson 2002]. 


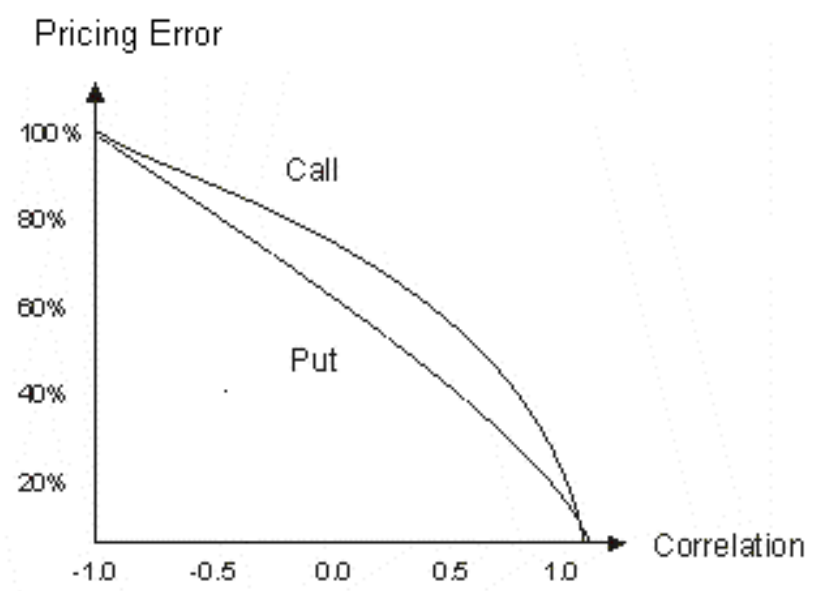

Figure 3. Pricing Errors as a Function Of Correlation

It is worth noticing that the above calculations present the point of view of the option holder (the one possessing the $\mathrm{X}$ asset and the put on $\mathrm{Y}$ ). However, if we look at the value of the put from the writer's point of view (for that purpose the portfolio " $\mathrm{X}$ - put $\mathrm{Y}$ " is created) we will see that the writer valuates the same put differently. The two values are often called upper hedging and lower hedging prices [Rouge 2000]. Prices of the put (shown in Figure 3) are derived from the "X - put Y" portfolio in the same fashion as it has been done with the "X+ put Y" portfolio; namely, with the "equal market price of risk" being the main rule.

The bid-ask spread that appears implies a range of possible put prices to be offered or demanded. The phenomenon is in accordance with the Hubalek and Schachermayer's theorem (compare Appendix 1) which for imperfect correlation in this case justifies any put price within the range between 0 and $\frac{5}{1+0.05}$ without violating the no-arbitrage principle. Similar behaviour of prices can be observed in a case of the call.

It is also worth repeating after Cochrane (Cochrane, Sao-Requejo 1996) that "when the bounds are large they quantify our ignorance. Payoffs that have better approximate hedges will have tighter bounds". 
The bid-ask spread concept obviously lies in the nature of any business transactions. A businessperson who would like to have certain flexibility (to grow, to abandon the business) tends to offer lower prices than the person that commits to provide such flexibility. The final price, lying within the bid-ask spread is a result of either negotiations or partial hedging. Bidask spread, even if transaction costs are not involved, emerges in a natural way in incomplete markets as a result of super-hedging applied by the writer and sub-hedging by the buyer of an option.

In general, we can consider portfolios of the form $X+\kappa \times$ put $Y$ (their values marked as $P_{\kappa}$ ), where $-1 \leq \kappa \leq 1$. When $\kappa$ runs from -1 to 1 the option prices calculated as $\left(P_{\kappa}-X(0)\right) \times \frac{1}{\kappa}$ fill the space inside the spindle in Figure 4 (the same procedure applies to a call).

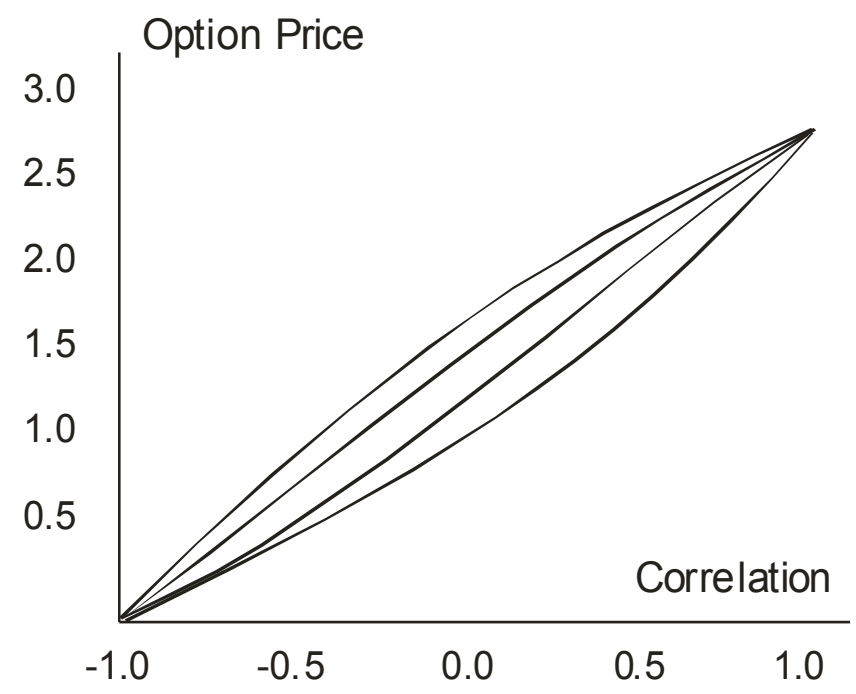

Figure 4. Bid ask spread for put 


\section{Conclusions}

The above examples indicate the complexity involved in real options valuation. It is tempting to apply financial options valuation techniques. It is also possible, provided there is a financial asset that is perfectly correlated with the underlying asset of the real option. However, imperfect correlation may lead to a substantial lack of precision in the valuations. In most cases we only have an approximate hedge. We can only calculate bounds, using a set of approximate hedges that are actually available.

An important argument against using the financial tools to real options is provided by Hubalek and Schachermayer. An exposition of the one step binomial counterpart of their result provided in this paper shows that any prices lying within the bounds between 0 and 4.762 for the put or 0 and 19.048 for the call are consistent with the no-arbitrage principle.

We propose a method based on corporate finance approach to valuation. The option price is obtained from the expected payoff of the business with options attached, with respect to the physical probability, discounted by the cost of capital. The discount rate is obtained as a result of assuming the invariance of market price of risk if an option is added to the underlying asset. We can valuate options for various correlation coefficients between the real and financial assets in one step discrete model. We have shown that the real option prices are increasing in correlation, meaning that the investor is willing to pay more when he is more likely to have a realistic hedge. The real option prices obtained are between 0 and 2.857 for the put and 0 and 7.619 for the call. The bid-ask prices resulting from using more general portfolios, involving option writers, also fit within the bounds. 
The approach we suggest may help overcome technical problems related to implementing real options. It shows that real options can be properly valuated if only the adequate measure of correlation between the involved assets is incorporated in the valuation model.

\section{Appendix 1}

We first recall the main result of [Hubalek 2001] concerned with arbitrage limits on price of real call option, and then prove its discrete single-step counterpart for a general derivative security.

Theorem (Hubalek and Schachermayer [Hubalek 2001]). Let $X(t), Y(t)$ be stochastic processes representing a real and a traded asset, respectively, $t \geq 0$, defined on some filtered probability space $\left(\Omega, F_{t}, F, P\right)$. Assume that they admit the Black-Scholes type representation

$$
\begin{aligned}
& d X(t)=\mu_{1} X(t) d t+\sigma_{1} X(t) d W_{1}(t), \\
& d Y(t)=\mu_{2} Y(t) d t+\sigma_{2} Y(t) d W_{2}(t),
\end{aligned}
$$

where the correlation between $W_{1}(t)$ and $W_{2}(t)$ is $\rho<1$.

Then for each positive real number $z$ there is a probability measure $Q$ equivalent to $P$ such that

1. $Y(t)$ is a martingale with respect to $Q$,

2. The Black-Scholes price of a real European call (written on $X$ with strike price $K$ and exercise time $T$ ) is $z$, that is

$$
z=e^{-r T} E_{Q}\left((X(T)-K)^{+}\right)
$$

As a consequence, the market consisting of the traded asset $Y(t)$, the call prices process

$$
C(t)=e^{-r(T-t)} E_{Q}\left((X(T)-K)^{+} \mid F_{t}\right),
$$


with $C(0)=z$, is arbitrage free.

Next we prove a version of this result for the single step quadrinomial model considered in this paper. We work with the general model introduced at the beginning of Section 3.1

Theorem. Let $X(t), Y(t), t=0,1$, represent real and traded asset, respectively, defined on a four-element probability space with probabilities $p_{i} \in(0,1), i=1,2,3,4$ such that

$$
\begin{aligned}
& X(1)= \begin{cases}X(0)\left(1+u_{X}\right) & \text { with prob. } p_{1}+p_{2} \\
X(0)\left(1+d_{X}\right) & \text { with prob. } p_{3}+p_{4}\end{cases} \\
& Y(1)= \begin{cases}Y(0)\left(1+u_{Y}\right) & \text { with prob. } p_{1}+p_{3} \\
Y(0)\left(1+d_{Y}\right) & \text { with prob. } p_{2}+p_{4}\end{cases}
\end{aligned}
$$

Denote by $p_{*}$ the risk neutral (martingale) probability for $Y$, that is $p_{*}=\frac{r-d_{Y}}{u_{Y}-d_{Y}}$ where $r$ is the risk free rate. Consider a claim $C$ contingent on the real asset $X$ with the payoff of the form

$$
\left\{\begin{array}{l}
C^{u}=f\left(X(0)\left(1+u_{X}\right)-K\right) \\
C^{d}=f\left(X(0)\left(1+d_{X}\right)-K\right)
\end{array}\right.
$$

Assume that $C^{u}>C^{d}$. Then for each

$$
z \in\left(\frac{C^{d}}{1+r}, \frac{C^{u}}{1+r}\right)
$$

there exists a quadruple $Q=\left(q_{1}, q_{2}, q_{3}, q_{4}\right)$ of $q_{i} \in(0,1), i=1,2,3,4$ such that

1. $Y$ is a martingale under $Q$, that is

$$
Y(0)=\frac{1}{1+r}\left(Y(0)\left(1+u_{Y}\right)\left(q_{1}+q_{3}\right)+Y(0)\left(1+d_{Y}\right)\left(q_{2}+q_{4}\right)\right)=\frac{1}{1+r} E_{Q}(Y(1))
$$

2. The price of a the contingent claim $C$ is

$$
z=\frac{1}{1+r}\left(C^{u}\left(q_{1}+q_{2}\right)+C^{u}\left(q_{3}+q_{4}\right)\right)=\frac{1}{1+r} E_{Q}\left((X(1)-K)^{+}\right)
$$


Conversely, if $z$ is outside the interval $\left(\frac{C^{d}}{1+r}, \frac{C^{u}}{1+r}\right)$, and it is the price of the claim, we have an arbitrage opportunity.

Proof. In order to obtain property 1 we have the following condition

$$
q_{1}+q_{3}=p_{*}
$$

For $Q$ to be a probability we must have

$$
q_{1}+q_{2}+q_{3}+q_{4}=1
$$

The formula in property 2 gives additional equation

$$
z=\frac{C^{u}}{1+r}\left(q_{1}+q_{2}\right)+\frac{C^{d}}{1+r}\left(q_{3}+q_{4}\right)
$$

Thus we have a system (1-3) of three equation with four variables. We seek a solution consisting of positive numbers:

$$
q_{i}>0, \quad i=1,2,3,4
$$

Solving (1) for $q_{1}$ and inserting into (2), (3) we get

$$
\begin{aligned}
& p_{*}+q_{2}+q_{4}=1 \\
& z=\frac{C^{u}}{1+r}\left(p_{*}-q_{3}+q_{2}\right)+\frac{C^{d}}{1+r}\left(q_{3}+q_{4}\right) .
\end{aligned}
$$

Adding conditions

$$
q_{i}>0, \quad i=2,3,4,
$$

$$
q_{3}<p_{*},
$$

we obtain a system $(1,5-8)$ equivalent to (1-4).

Next we solve (5) for $q_{2}$ and insert in (6) to get

$$
z=\frac{C^{u}}{1+r}\left(1-q_{3}-q_{4}\right)+\frac{C^{d}}{1+r}\left(q_{3}+q_{4}\right) .
$$


If a solution satisfying

$$
\begin{aligned}
& q_{i}>0, \quad i=3,4 \\
& q_{3}<p_{*}, \quad q_{4}<1-p_{*}
\end{aligned}
$$

can be found, this guarantees the existence of a quadruple $\left(q_{1}, q_{2}, q_{3}, q_{4}\right)$ with the desired properties (and also conversely, as the argument shows).

Now, it is elementary to see that this is equivalent to the condition $\left(^{*}\right)$ on $z$ (the line determined by (9) in the $\left(q_{3}, q_{4}\right)$ - plane intersects the rectangle (10), (11) if and only if $(*)$ holds), which completes the proof.

Remark. In Hubalek-Schachermayer theorem there is no restriction on $z$ in contrast to the discrete case. This is explained by the fact that in the continuous framework the lognormal distributions of the asset prices allow extreme values (though with small probabilities). These values, unlikely but possible, do not allow arbitrage even if the real option is seemingly seriously mispriced. In such a case there would be large probability, but not certainty, that an arbitrage-like profit would be possible.

\section{Appendix 2}

The option value for various correlations (which is equivalent to certain values of $q$, $\rho=4 \times q-1$ ), can be easily found from the equation below .

$$
0,2=\frac{k_{P}-0,05}{\sigma_{P}}
$$


where $k_{P}, \sigma_{P}$ are expected return and standard deviation of the portfolio $P$, respectively., and 0.2 is the market price of risk for $X$. If we denote the price of the option by $x$ we get

$$
\begin{gathered}
k_{P}=\frac{5 \times q+7,5-x}{100+x} \\
\sigma_{P}=\left(q\left(t-k_{P}\right)^{2}+(0,5-q)\left(t-k_{P}\right)^{2}+(0,5-q)\left(\frac{-5-x}{100+x}-k_{P}\right)^{2}+q\left(-\frac{x}{100+x}-k_{P}\right)^{2}\right)^{0,5}
\end{gathered}
$$

where $t=\frac{20-x}{100+x}$. The solution to the equation with respect to $x$ is

$$
x=\frac{-0,01814}{43+2 \times q} \times\left(-504 \times q^{2}-11466 \times q-5775+\frac{130 \times c \times q^{2}+5395 \times c \times q+57750 \times c}{b}\right)
$$

where

$$
\begin{aligned}
& a=\sqrt{46225-10048 \times q^{2}-25284 \times q-16 \times q^{4}-752 \times q^{3}}, \\
& b=4620000+10400 \times q^{2}+431600 \times q, \\
& c=10500-1680 \times q^{2}-6720 \times q+2100 \times a .
\end{aligned}
$$

\section{References}

Amram M., N. Kulatilaka. (1999): Uncertainty: The New Rules for Strategy. The Journal of Business Strategy 20 (3)

Amram M., N. Kulatilaka. (2000): Strategy and Shareholder Value Creation: The Real Options Frontier. Journal of Applied Corporate Finance13: 8-21

Bielecki T.R., M. Jeanblanc, M. Rutkowski. (2003): Pricing and hedging of credit risk: replication and mean-variance analysis. Ams-Ims-Siam Joint Summer Research 
Conference on Mathematics of Finance. June 22-26, 2003. Snowbird, Utah (George Yin and Qing Zhang eds.) American Mathematical Society.

Black F., M. Scholes.(1973): The Pricing of Options and Corporate Liabilities. Journal of Political Economy 81: 637-659

Boer F.P. (2000): Valuation of Technology Using Real Options. Research Technology Management. July/August 2000

Boyle P., T. Wang. (2001): Pricing of New Securities in an Incomplete Market: the Catch 22 of No-arbitrage Pricing. Mathematical Finance 11 (3): 267 - 284

Cochrane J.H., J. Sao-Requejo. (1996): Beyond arbitrage: "good-deal” asset price bounds in incomplete markets. NBER Working Paper Series. Working Paper 5489.

Constantinides G. (1978): Market Risk Adjustment in Project Valuation. Journal of Finance 33 (2): 603-616

Copeland T., V. Antikarov V. (2000): Real Options. A Practitioner's Guide, Texere

Cotner J.S., H.D. Fletcher. (2000): Computing the cost of capital for privately held firms. American Business Review 18(2): 27-33.

Cox J., S. Ross, M. Rubinstein. (1979): Option Pricing: A Simplified Approach. Journal of Financial Economics 7: 229-263

Cox J., S. Ross. (1976): The Valuation of Options for Alternative Stochastic Processes. Journal of Financial Economics 3 (1/2): 229-263

Dixit A.K., R.S. Pindyck. (1994): Investment under Uncertainty. Princeton NJ: Princeton University Press

Fernandez P. (2002): Valuation methods and Shareholder Value Creation. San Diego: Academic Press.

Hansen L.P., R. Jagannathan. (1991): Implications for Security Market Data for Models of Dynamic Economies. Journal of Political Economy 99: 225-262. 
Henderson V., D.G.Hobson. (2002): Real options with constant relative risk aversion. Journal of Economic Dynamics \& Control 27 (2): 329-355.

Herath H.S.B., C.S. Park. (2001): Exploiting Uncertainty - Investment Opportunities as Real Options: A New Way of Thinking in Engineering Economics. The Engineering Economist 45 (1): 2-36

Hubalek F., W. Schachermayer. (2001): The Limitations of No-Arbitrage Arguments for Real Options. International Journal of Theoretical and Applied Finance 4(2): 361-373

Hull J.C. (1989): Options, Futures and Other Derivatives. Prentice Hall

Kellog D., J.M. Charnes. (2000): Real Options Valuation for a Biotechnology Company. Financial Analysts Journal. May/ June 2000: 76-84

Kemna A. (1993): Case Studies on Real Options. Financial Management. Autumn

Merton R.C. (1973): Theory of Rational Option Pricing. Bell Journal of Economics and Management Sciences 4 (1): 141-183

Rouge R., N. Karoui. (2000): Pricing via Utility Maximization and Entropy. Mathematical Finance 10 (2): 259-276

Trigeorgis L. (1999): Real Options ( $4^{\text {th }}$ Ed). The MIT Press

University of Maryland. (2003): Round Table on Real Options and Corporate Practice. Journal of Applied Corporate Finance15 (2) 